\title{
Outcomes of Intradetrusor Onabotulinum Toxin A Therapy in Overactive Bladder Refractory to Sacral Neuromodulation
}

\author{
Hamilton Trinh ${ }^{1}$, Vicki Irish², Mireya Diaz ${ }^{2}$, Humphrey Atiemo \\ ${ }^{1}$ Wayne State School of Medicine, Detroit, MI, USA \\ ${ }^{2}$ Vattikuti Urology Institute, Henry Ford Hospital, Detroit, MI, USA
}

Purpose: Intradetrusor onabotulinum toxin A (BTXA) and sacral neuromodulation (SNM) are effective third-line therapies for overactive bladder $(\mathrm{OAB})$. We aimed to measure the outcomes of BTXA for treatment of OAB refractory to initial SNM and identify patient characteristics associated with these outcomes.

Methods: This retrospective cohort study included patients who failed to respond to initial SNM treatment for OAB and subsequently received BTXA at a single provider's clinic between January 2013 and December 2016. Treatment successes were defined as patients willing to continue BTXA or who found symptom relief whereas treatment failures discontinued BTXA due to adverse effects or lack of symptom relief. Symptoms and patient-reported outcomes on validated questionnaires were compared before the initial BTXA trial to 2 months after the last BTXA treatment. The SNM failure BTXA groups were also compared to BTXA SNM naïve groups.

Results: Of 18 patients who received BTXA after failed SNM treatment, 7 (39\%) achieved treatment success. Successfully treated patients demonstrated decreased urinary frequency from a median 11 voids/day pre-BTXA to 8 voids/day with BTXA $(\mathrm{P}=0.042)$. Patients whose treatment failed reported increased complaints of a weak urinary stream $(\mathrm{P}=0.03)$ and higher frequency of straining to urinate $(\mathrm{P}=0.016)$ than the successful treatment group pre-BTXA. Compared to BTXA patients without prior SNM, the odds of failing BTXA after initial SNM were 3.6 times higher $(\mathrm{P}=0.016)$.

Conclusions: BTXA appears effective for OAB refractory to SNM, although the success rate is lower compared to BTXA patients without SNM exposure.

Keywords: Urinary bladder, overactive; Onabotulinum toxin A; Sacral neuromodulation

- Research Ethics: The study was performed according to the Helsinki Declaration and approved by the Institutional Review Board (IRB) of Henry Ford Health System (IRB No. 11369). Written informed consent was obtained from all subjects.

- Conflict of Interest: No potential conflict of interest relevant to this article was reported.

\section{INTRODUCTION}

Overactive bladder $(\mathrm{OAB})$ is a condition of urinary urgency and frequency with or without incontinence and often nocturia [1]. It affects $30 \%$ of women and $16 \%$ of men in the United States and has a significant impact on patient quality of life as well as health care resources [2-6]. First-line therapy for OAB includes behavioral modifications such as fluid restriction, dietary management, and timed voiding [7]. If behavioral modifications fail to control symptoms, second-line measures include pelvic floor physical therapy and oral anticholinergics and beta-3 agonists $[7,8]$. These therapies may fail due to poor com-

Corresponding author: Humphrey Atiemo (iD https://orcid.org/0000-0002-9784-655X Vattikuti Urology Institute, Henry Ford Hospital, 2799 West Grand Blvd, Detroit, MI 428202, USA

E-mail: hatiemo1@hfhs.org / Tel: +1-313-817-4712 / Fax: +1-313-916-9539

Submitted: January 23, 2019 / Accepted after revision: June 4, 2019

This is an Open Access article distributed under the terms of the Creative Commons Attribution Non-Commercial License (http://creativecommons.org/licenses/by-nc/4.0/) which permits unrestricted non-commercial use, distribution, and reproduction in any medium, provided the original work is properly cited. 
pliance with pelvic floor physical therapy or side effects associated with medical therapy [9]. Sacral neuromodulation (SNM) with Interstim (Medtronic, Minneapolis, MN, USA) and intradetrusor onabotulinum toxin A (BTXA) injections are well established third-line therapies in the OAB treatment pathway [7]. BTXA has a success rate of $70 \%-80 \%$ after the initial trial, and SNM has a success rate of $60 \%-70 \%$ over a mean of 53 months $[10,11]$. It is common practice to offer $\mathrm{OAB}$ patients the choice of either SNM or BTXA when considering third-line therapy. While selection of an initial third-line treatment is an individualized process based on patient preferences and comorbidities, patients who fail an initial third-line therapy may be offered a subsequent third-line therapy. Studies have reported a 70\% success rate of SNM in OAB patients who failed initial BTXA trials, similar to the effectiveness of BTXA naïve SNM patients $[12,13]$. While BTXA has been well characterized as an initial third-line treatment, there is a lack of data on the outcomes of BTXA in OAB patients who failed SNM and on predictors associated with its outcome.

This study aimed to measure the outcomes of BTXA for the treatment of $\mathrm{OAB}$ refractory to initial SNM through clinical urinary symptoms, patient-reported outcomes on validated questionnaires, and BTXA continuation rates. We hypothesized that the continuation rate of BTXA as a third-line therapy would be similar to that of BTXA patients with no prior SNM exposure, and that BTXA after failed response to SNM for OAB would produce significant improvement as noted by patient-reported urinary symptoms, incontinence severity, and quality of life using validated questionnaires. We also sought to identify characteristics prior to BTXA rescue therapy associated with these outcomes.

\section{MATERIALS AND METHODS}

This retrospective cohort study identified all patients treated with BTXA and those treated with SNM from January 2013 to December 2016 in a single urologist's clinic. Only patients with a clinical diagnosis of refractory $\mathrm{OAB}$ with urodynamic detrusor overactivity were eligible for inclusion. The study was approved by the hospital's Institutional Review Board.

Of the SNM group, each patient's OAB symptoms were treated initially with either stage I or stage II SNM. Patients who failed SNM were then given an option of BTXA for their symptoms. Failure of SNM therapy was defined as ineffective symptom control with less than $50 \%$ symptom improvement during stage I and after stage II, inability to maintain greater than 50\% improvement in baseline symptoms, or for patients with urinary frequency, more than 8 voids per day, adverse effects such as pain, or patient dissatisfaction within stage 1 or $2[1,14]$. All SNM failure patients underwent urodynamic evaluation prior to subsequent BTXA treatment. Urodynamic bladder capacity and average volume at the time of uninhibited detrusor contractions were recorded.

Of the BTXA group, patients included those exposed and not exposed to SNM before BTXA treatment. BTXA patients who had prior SNM exposure were divided into BTXA treatment success and treatment failure for comparison. We defined treatment success as patient willingness to continue BTXA within the 4-year study period or who found subjective symptom relief with BTXA. We defined treatment failure as patient discontinuation of BTXA within the study period due to subjective lack of relief from symptoms, lack of follow-up, or adverse side effects such as urinary retention. Patients in the BTXA treatment success and failure groups were also compared to refractory OAB patients who had not been exposed to SNM prior to BTXA treatment.

All patients had been asked to complete the previously validated American Urological Association Symptom Score (AUASS) questionnaire and Michigan Incontinence Symptom Index (M-ISI) across the study period. Daily pad usage, daytime voiding frequency, AUASS scores (symptoms and quality of life) and individual question scores, and M-ISI severity and bother scores were compared before BTXA and 2 months after the latest BTXA treatment to assess voiding patterns and outcomes for treatment success and failure groups $[15,16]$. These outcomes served to define BTXA efficacy in OAB patients who failed initial SNM and to determine factors that might be associated with therapy outcomes.

Descriptive statistics were utilized to determine BTXA continuation rates in patients with prior SNM treatment. The chi-square test with significance set at $\mathrm{P}<0.05$ was used to compare treatment successes and failures in the BTXA group who had prior SNM exposure and the BTXA group without SNM exposure. Wilcoxon signed-rank test was used to compare voiding patterns and patient-reported responses to questionnaires prior to and 2 months after BTXA. The Mann-Whitney U-test was used to compare age, voiding patterns, and questionnaire response between the treatment success and failure groups prior to the initial BTXA treatment. All statistical analyses were carried out with IBM SPSS Statistics ver. 24.0 (IBM Co., Armonk, NY, USA). 


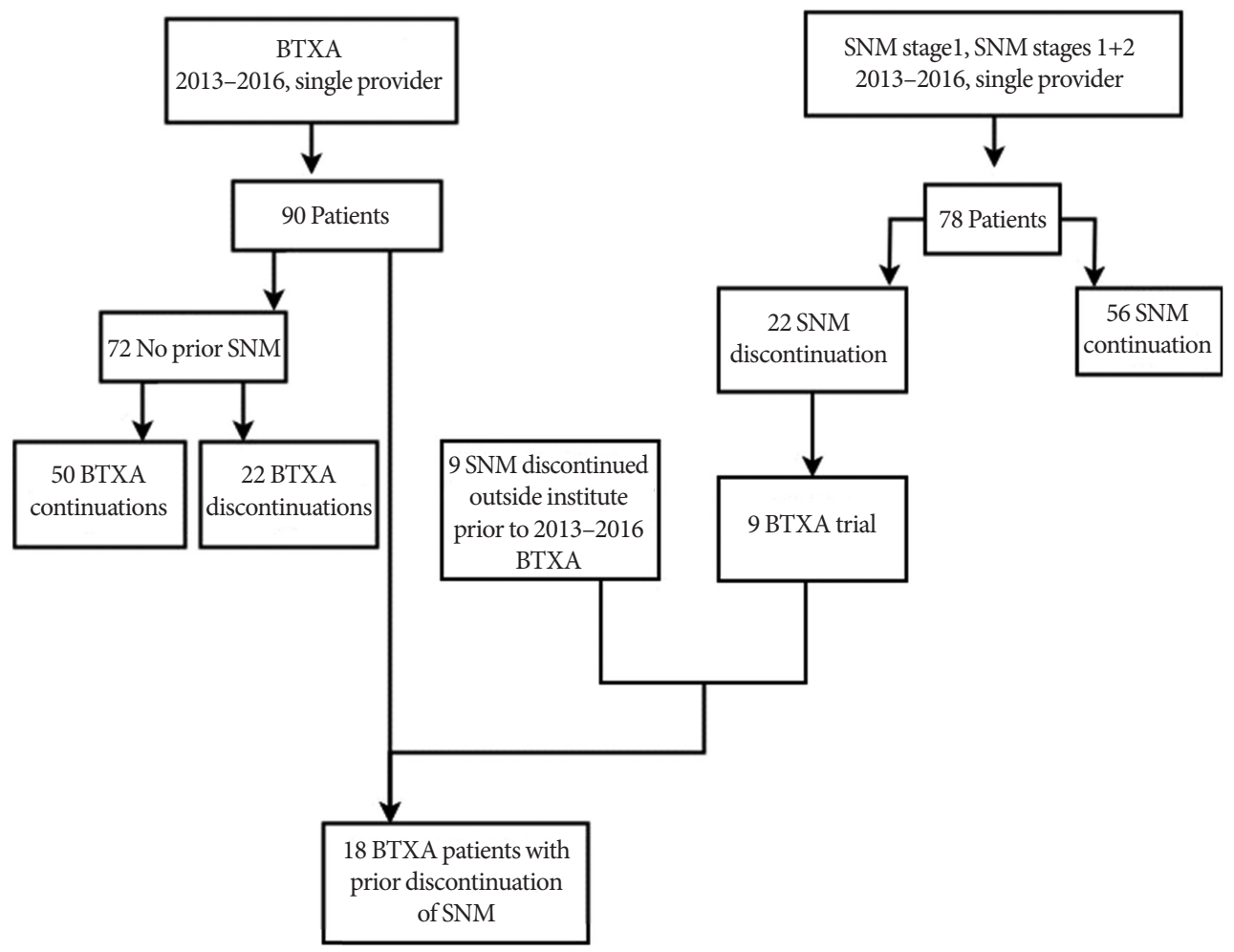

Fig. 1. Derivation of study cohort. BTXA, intradetrusor onabotulinum toxin A; SNM, sacral neuromodulation.

\section{RESULTS}

Of all refractory OAB patients identified, 78 patients had SNM and 90 patients received BTXA (Fig. 1). Of the 78 SNM patients, $56(71.8 \%)$ met criteria for successful SNM treatment. Of the 90 BTXA patients, 72 patients had no prior SNM exposure with $50(69.4 \%)$ meeting criteria for successful BTXA treatment (Fig. 1). Of note, 3 of the 72 had prior posterior tibial nerve stimulation therapy, with 2 successfully treated with subsequent BTXA.

Of the 18 patients in the BTXA treatment group with prior failed SNM, 7 (38.9\%) met criteria for successful BTXA treatment. Nine of the 18 patients had received their SNM trial from an outside institution. On the AUASS questionnaire pre-BTXA, the treatment failure group had statistically significant increased frequency of weak urine streams (AUASS question $5 ; \mathrm{P}=0.03$, Mann-Whitney U-test) and increased frequency of straining to void (AUASS question 6; $\mathrm{P}=0.016$, Mann-Whitney U-test) compared to the treatment success group (Table 1). No statistically significant differences were noted between the BTXA treatment success and failure groups in baseline characteristics of age, bladder capacity, mean uninhibited detrusor contraction volume, daytime voiding frequency, nocturia, daily pad usage, AUASS symptoms and quality of life scores, M-ISI severity and bother scores, and AUASS questions 1-4 and 7 (Table 1).

Daytime voiding frequency for the BTXA treatment success group had a statistically significant decrease from a median 11 voids/day before initial BTXA treatment to 8 voids/day after BTXA ( $\mathrm{P}=0.042$, Wilcoxon signed-rank test) (Table 2). The treatment failure group showed no significant change in daytime voiding frequency. Daily pad usage, AUASS symptom and quality of life and individual question scores, and M-ISI severity and bother scores showed no significant changes in the treatment success group before versus after BTXA (Table 2). Similarly, the baseline parameters for the treatment failure group had no significant changes before versus after BTXA (Table 2).

A comparison of the 18 BTXA patients who had failed prior SNM and the 72 BTXA patients with no prior SNM showed that prior SNM treatment was associated with a statistically significant risk of BTXA failure, $\chi^{2}(1)=5.8$, odds ratio $=3.6(95 \%$ confidence interval, $1.23-10.42 ; \mathrm{P}=0.016$, chi-square test).

Ten of the 18 BTXA patients with prior SMN had a neuro- 
Table 1. Baseline characteristics of patients with overactive bladder refractory to SNM

\begin{tabular}{|c|c|c|c|}
\hline Characteristic $^{\text {a) }}$ & Treatment success & Treatment failure & 2-tailed P-value ${ }^{\text {b) }}$ \\
\hline No prior SNM & 50 & 22 & \\
\hline Male & 7 & 4 & \\
\hline Female & 43 & 18 & \\
\hline Prior SNM & 7 & 11 & \\
\hline Male & 4 & 4 & \\
\hline Female & 3 & 7 & \\
\hline Age (yr) & $74(50-80)$ & $77(62-80)$ & 0.436 \\
\hline Bladder capacity (mL) & $322(97-546)$ & $210(144-339)$ & 0.618 \\
\hline Mean UDC volume (mL) & $266(97-321)$ & $178(131-364)$ & 0.874 \\
\hline Daytime voiding frequency & $11(6-15)$ & $8(4-17)$ & 0.056 \\
\hline Nocturia & $2(0-5)$ & $3(0-7)$ & 0.804 \\
\hline Daily pad usage & $2(0-10)$ & $2(0-20)$ & 0.854 \\
\hline AUASS & $15(0-24)$ & $24(8-32)$ & 0.130 \\
\hline Quality of life & $5(2-6)$ & $4(1-6)$ & 0.543 \\
\hline M-ISI severity & $21(0-30)$ & $21(3-32)$ & 0.685 \\
\hline M-ISI bother & $6(0-8)$ & $5(0-8)$ & 0.441 \\
\hline AUAQ1 & $1(0-5)$ & $4(1-5)$ & 0.059 \\
\hline AUAQ2 & $5(0-5)$ & $5(2-5)$ & 0.896 \\
\hline AUAQ3 & $3(0-4)$ & $3(1-5)$ & 0.375 \\
\hline AUAQ4 & $5(0-5)$ & $4(0-5)$ & 0.541 \\
\hline AUAQ5 & $0(0-5)$ & $4(0-5)$ & 0.030 \\
\hline AUAQ6 & $0(0-1)$ & $2(0-5)$ & 0.016 \\
\hline AUAQ7 & $2(0-5)$ & $3(1-5)$ & 0.437 \\
\hline
\end{tabular}

Values are presented as number or median (range).

SNM, sacral neuromodulation; UDC, uninhibited detrusor contractions; AUASS, American Urological Association Symptom Score; M-ISI, Michigan Incontinence Severity Index; AUAQ1-7, American Urological Association Symptom Score questions.

${ }^{\text {a) }}$ Sex, age, bladder capacity, mean UDC volume, daytime voiding frequency, nocturia, pad usage, and questionnaire scores listed are for the 18 pa-

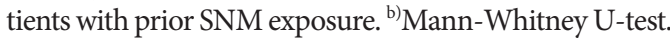

logical disorder: 3 had a history of spinal degeneration defined as either spinal stenosis or lumbosacral radiculopathy, another 3 had a history of cerebrovascular accident, 1 had both cerebrovascular accident and spinal stenosis, 1 multiple sclerosis, 1 cerebral palsy, and 1 had both Parkinson disease and lumbosacral radiculopathy (Table 3). BTXA was successful in 4 of these patients: 1 with multiple sclerosis, 2 with spinal degeneration, and 1 with cerebrovascular accident (Table 3 ).

\section{DISCUSSION}

This study assesses the results of BTXA therapy after failure of initial SNM in OAB patients. While SNM is an effective thirdline therapy for $\mathrm{OAB}$, clinical factors causing its failure and dis- continuation may include technical issues during stage I such as improper lead placement, lack of adequate response to stage I, central nervous system pathology, and progression of disease resulting in $\mathrm{OAB}$ refractory to $\mathrm{SNM}$ after stage II. Based on our study criteria, only $39 \%$ (7 of 18) achieved treatment success with BTXA after SNM failure. No other studies to date have reported outcomes in this subgroup of patients. While the BTXA treatment success rate in our non-SNM cohort was similar to the $70 \%-80 \%$ initial success rate reported for BTXA patients without prior SNM [11], the BTXA treatment success rate in the failed SNM cohort was much lower. The odds ratio of 3.6 in our cohort indicates that failing initial SNM is associated with a much higher risk of failing subsequent BTXA. Previous studies on $\mathrm{OAB}$ refractory to an initial third-line therapy showed SNM 
Table 2. Efficacy and quality of life after treatment with BTXA in patients with overactive bladder refractory to SNM

\begin{tabular}{|c|c|c|c|c|}
\hline \multirow{2}{*}{ Characteristic } & \multicolumn{2}{|c|}{ Treatment success } & \multicolumn{2}{|c|}{ Treatment failure } \\
\hline & Median (range) & 2-tailed paired P-value ${ }^{\text {a) }}$ & Median (range) & 2-tailed paired P-value ${ }^{a)}$ \\
\hline Daytime voiding frequency & $8(5-11)$ & 0.042 & $6(0-20)$ & 0.916 \\
\hline Daily pad usage & $1(0-4)$ & 0.059 & $1(0-8)$ & 0.102 \\
\hline AUASS & $11(1-20)$ & 0.343 & $22(6-35)$ & 0.767 \\
\hline Quality of life & $2(1-6)$ & 0.063 & $5(1-6)$ & 0.785 \\
\hline M-ISI severity & $12(0-29)$ & 0.136 & $11(2-32)$ & 0.144 \\
\hline M-ISI bother & $3(0-8)$ & 0.063 & $4(0-8)$ & 0.854 \\
\hline Nocturia & $3(0-4)$ & 1.000 & $2(1-7)$ & 0.492 \\
\hline AUAQ1 & $0(0-1)$ & 0.257 & $4(1-5)$ & 0.317 \\
\hline AUAQ2 & $3(0-5)$ & 0.157 & $4(0-5)$ & 0.336 \\
\hline AUAQ3 & $0(0-4)$ & 0.102 & $4(1-5)$ & 0.480 \\
\hline AUAQ4 & $2(0-5)$ & 0.498 & $4(1-5)$ & 0.414 \\
\hline AUAQ5 & $0(0-4)$ & 0.180 & $3(0-5)$ & 0.236 \\
\hline AUAQ6 & $0(0-5)$ & 0.317 & $3(0-5)$ & 0.450 \\
\hline AUAQ7 & $3(1-5)$ & 0.131 & $2(1-5)$ & 0.357 \\
\hline
\end{tabular}

BTXA, intradetrusor onabotulinum toxin A; SNM, sacral neuromodulation; AUASS, American Urological Association Symptom Score; M-ISI, Michigan Incontinence Severity Index; AUAQ1-7, American Urological Association Symptom Score questions.

${ }^{a)}$ Wilcoxon signed-rank test.

Table 3. Risk factors for neurogenic bladder

\begin{tabular}{lcc}
\hline Characteristic & Treatment success (n) & Treatment failure (n) \\
\hline Spinal degeneration ${ }^{\text {a) }}$ only & 2 & 1 \\
Cerebrovascular accident only & 1 & 2 \\
Cerebrovascular accident+spinal stenosis & 0 & 1 \\
Multiple sclerosis & 1 & 0 \\
Cerebral palsy & 0 & 1 \\
Parkinson disease+lumbosacral radiculopathy & 0 & 1 \\
\hline
\end{tabular}

${ }^{\text {a) }}$ Spinal stenosis or lumbosacral radiculopathy.

after initial BTXA treatment to be just as effective as SNM in patients who had no prior BTXA $[12,13]$. Unlike the other studies which included mostly women, our study was nearly equally divided in gender ( 8 men, 10 women). We used the AUASS questionnaire to assess pre- and post-BTXA outcomes as it has been shown to be equally useful in evaluating responses to treatment for urinary symptoms for both men and women [17].

The mechanism of BTXA inhibits signaling peripherally in the bladder whereas SNM regulates the central nervous system pathway associated with micturition. The most widely accepted explanation for BTXA is the toxin's cleavage and deactivation of SNAP-25, a T-SNARE protein needed for acetylcholinergic neurotransmitter vesicle fusion at synaptic junctions. Cholinergic synaptic transmission for afferent and efferent nerve endings in the bladder is thus reduced and results in decreased sensation of urge and amount of detrusor contractions $[18,19]$. The hypothesized effect of SNM is through pulsed electrical stimulation of the roots of the S3 and S4 pelvic and pudendal nerves [20]. The pulses travel to the central nervous system and modulate reflex pathways within the pontine and spinal micturition centers that control the emptying and storage phases of the micturition cycle [21,22]. Because the mechanisms of SNM and BTXA differ and thus appear to be independent of each other, we hypothesized that prior SNM would not affect the success of 
BTXA treatment and, therefore, the BTXA success rate for patients refractory to SNM would be similar to BTXA for patients without prior SNM. The lower success rate in our study $39 \%$ for BTXA after failed SNM versus $69 \%$ for BTXA without $\mathrm{SNM}$ ) might be explained by potentially more severe baseline comorbidities affecting bladder and central nervous system function in our patient cohort. Of note, 10 of our 18 patients had a neurological disorder; BTXA was successful in 4 of the 10 patients. Despite the heterogeneity of the neurological disorders, these disorders have all been associated with an increased risk of detrusor overactivity $[1,23]$. We hypothesize that the 4 patients with central neurological disorders may have had success with BTXA due to BTXA's mechanism of action on parasympathetic nerves within the detrusor muscle. These patients may also have lowered their expectations for symptom improvement after failing initial SNM. This example demonstrates the importance of patient counseling on the use of a subsequent third-line option for OAB after initial third-line therapy failure. While the mechanisms of action of SNM and BTXA are different, this population with neurogenic detrusor overactivity may be inherently more refractory to third-line therapy for OAB.

The only voiding parameter in our study data that showed statistically significant improvement for the BTXA success group was median daytime voiding frequency, which showed a $27 \%$ improvement. As AUASS scores did not change significantly in either the success or failure group before versus after BTXA therapy, we were not able to identify additional reasons that lead patients to continue or discontinue BTXA treatment. It may be possible that patients in the treatment success group continued BTXA in the hope that subsequent BTXA injections would improve their urinary symptoms.

Analysis of individual question scores on the AUASS questionnaire showed statistically significant increases in frequency of straining to void and frequency of weak urine stream before the initial BTXA trial in the treatment failure group compared to the treatment success group. As urinary retention is a side effect of BTXA therapy, OAB patients bothered by symptoms of straining to void and weak stream may find these symptoms increased with BTXA and thus are less likely to find relief. Low urinary bladder compliance and capacity and bladder wall fibrosis are other factors associated with poor response to BTXA therapy [24]. Given that a significant number of our patients had neurological comorbidities, these factors may explain the increased BTXA discontinuation in our study.

Limitations of this study are the small sample size and the heterogeneity of the patient population reducing the ability of the study to be generalized. Due to the high success rate of SNM, identification of a large SNM failure group would require a multicenter randomized trial between SNM and BTXA. Of our 78 SNM patients, 22 failed SNM and only 9 of these proceeded to BTXA. An additional 9 patients had failed SNM from an outside institution. Similarly, other studies looking at the effectiveness of SNM after initial BTXA failure for OAB have been limited by small sample size also due to the high effectiveness of BTXA $[12,13]$. Many patients refractory to SNM chose not to proceed to a second third-line treatment or were concerned with the risk of urinary retention associated with BTXA. Another limitation of this study is our 4-year time frame. Extending the time frame may demonstrate an even greater decrease in BTXA success rate, given the significant reported discontinuation rate of BTXA over the long term [25-27].

No guidelines exist for choosing one third-line therapy over the other for OAB [28]. While the efficacy of BTXA compared to SNM is debated, an individualized approach based on clinical judgment and patient factors may be best in choosing an initial third-line therapy [29]. Both BTXA and SNM are effective third-line therapies for OAB. Our study shows that BTXA may be considered as another therapy worth trying after failure of initial SNM before returning to medical therapy, stopping all therapies and living with the symptoms, or considering augmentation cystoplasty. However, the BTXA discontinuation rate is higher due to lower efficacy compared to SNM naïve patients.

With increased usage of SNM and BTXA for refractory $\mathrm{OAB}$, more patients will become refractory to their initial thirdline treatment. This study measured the effectiveness of BTXA for OAB patients who failed and discontinued initial SNM. A history of SNM failure was associated with a threefold risk of failed subsequent BTXA injection. Additionally, a history of weak urine stream and increased straining to void on validated questionnaire scores were associated with higher failure of BTXA. To our knowledge, this is the first study to investigate the management of this complex patient population with evidence showing reduced but appreciable efficacy in the use of BTXA after SNM failure.

\section{AUTHOR CONTRIBUTION STATEMENT}

- Full access to all the data in the study and takes responsibility for the integrity of the data and the accuracy of the data analy- 
sis: $H T, V I, M D, H A$

- Study concept and design: $H T, V I, M D, H A$

- Acquisition of data: $H T, V I, M D, H A$

- Analysis and interpretation of data: $H T, V I, M D, H A$

- Drafting of the manuscript: HT, HA

- Critical revision of the manuscript for important intellectual content: HT, HA

- Statistical analysis: $H T, M D, H A$

- Administrative, technical, or material support: HT, VI, MD,

$H A$

-Study supervision: $H T, V I, M D, H A$

\section{REFERENCES}

1. South M, Karram MM. Overactive bladder syndrome and nocturia. In: Walters MD, Karram MM, editors. Urogynecology and reconstructive pelvic surgery. 4th ed. Philadelphia (PA): Saunders; 2015. p. 513-41.

2. Coyne KS, Sexton CC, Bell JA, Thompson CL, Dmochowski R, Bavendam T, et al. The prevalence of lower urinary tract symptoms (LUTS) and overactive bladder (OAB) by racial/ethnic group and age: results from OAB-POLL. Neurourol Urodyn 2013;32:230-7.

3. Ju R, Garrett J, Wu JM. Anticholinergic medication use for female overactive bladder in the ambulatory setting in the United States. Int Urogynecol J 2014;25:479-84.

4. Milsom I, Kaplan SA, Coyne KS, Sexton CC, Kopp ZS. Effect of bothersome overactive bladder symptoms on health-related quality of life, anxiety, depression, and treatment seeking in the United States: results from EpiLUTS. Urology 2012;80:90-6.

5. Chu CM, Ghetti C. Procedure-based management of urgency urinary incontinence in the older woman. Curr Geriatr Rep 2017;6: 98-113.

6. Jayadevappa R, Chhatre S, Newman DK, Schwartz JS, Wein AJ. Association between overactive bladder treatment and falls among older adults. Neurourol Urodyn 2018;37:2688-94.

7. Gormley EA, Lightner DJ, Faraday M, Vasavada SP; American Urological Association; Society of Urodynamics, Female Pelvic Medicine. Diagnosis and treatment of overactive bladder (nonneurogenic) in adults: AUA/SUFU guideline amendment. J Urol 2015;193:1572-80.

8. Zahner PM, Giusto LL, Goldman HB. What is the role of additional pharmacotherapy and neuromodulation in patients with marginal benefit from botulinum toxin injection? Curr Urol Rep 2018; 19:91.

9. Glinski RW, Siegel S. Refractory overactive bladder: beyond oral anticholinergic therapy. Indian J Urol 2007;23:166-73.

10. Al-zahrani AA, Elzayat EA, Gajewski JB. Long-term outcome and surgical interventions after sacral neuromodulation implant for lower urinary tract symptoms: 14-year experience at 1 center. J Urol 2011;185:981-6.

11. Denys P, Le Normand L, Ghout I, Costa P, Chartier-Kastler E, Grise P, et al. Efficacy and safety of low doses of onabotulinumtoxi$\mathrm{nA}$ for the treatment of refractory idiopathic overactive bladder: a multicentre, double-blind, randomised, placebo-controlled doseranging study. Eur Urol 2012;61:520-9.

12. Smits MA, Oerlemans D, Marcelissen TA, Van Kerrebroeck PE, De Wachter SG. Sacral neuromodulation in patients with idiopathic overactive bladder after initial botulinum toxin therapy. J Urol 2013;190:2148-52.

13. Hoag N, Plagakis S, Pillay S, Edwards AW, Gani J. Sacral neuromodulation for refractory overactive bladder after prior intravesical onabotulinumtoxinA treatment. Neurourol Urodyn 2017;36:137781.

14. Siegel S, Noblett K, Mangel J, Griebling TL, Sutherland SE, Bird ET, et al. Three-year follow-up results of a prospective, multicenter study in overactive bladder subjects treated with sacral neuromodulation. Urology 2016;94:57-63.

15. Barry MJ, Fowler FJ Jr, O’Leary MP, Bruskewitz RC, Holtgrewe HL, Mebust WK, et al. The American Urological Association symptom index for benign prostatic hyperplasia. The Measurement Committee of the American Urological Association. J Urol 1992;148: 1549-57.

16. Suskind AM, Dunn RL, Morgan DM, DeLancey JO, McGuire EJ, Wei JT. The Michigan Incontinence Symptom Index (M-ISI): a clinical measure for type, severity, and bother related to urinary incontinence. Neurourol Urodyn 2014;33:1128-34.

17. Rivas DA, Chancellor MB. Utility of the American Urological Association symptom index in the diagnosis of women with voiding dysfunction. Int Urogynecol J 1994;5:202-7.

18. Lawrence GW, Aoki KR, Dolly JO. Excitatory cholinergic and purinergic signaling in bladder are equally susceptible to botulinum neurotoxin a consistent with co-release of transmitters from efferent fibers. J Pharmacol Exp Ther 2010;334:1080-6.

19. Malde S, Fry C, Schurch B, Marcelissen T, Averbeck M, Digesu A, et al. What is the exact working mechanism of botulinum toxin $\mathrm{A}$ and sacral nerve stimulation in the treatment of overactive bladder/detrusor overactivity? ICI-RS 2017. Neurourol Urodyn 2018;37(S4):S108-16.

20. Leng WW, Chancellor MB. How sacral nerve stimulation neuromodulation works. Urol Clin North Am 2005;32:11-8. 
21. Abello A, Das AK. Electrical neuromodulation in the management of lower urinary tract dysfunction: evidence, experience and future prospects. Ther Adv Urol 2018;10:165-73.

22. Amend B, Matzel KE, Abrams P, de Groat WC, Sievert KD. How does neuromodulation work. Neurourol Urodyn 2011;30:762-5.

23. Aponte MM, Nitti VW. Voiding dysfunction and urinary retention. In: Walters MD, Karram MM, editors. Urogynecology and reconstructive pelvic surgery. 4th ed. Philadelphia (PA): Saunders; 2015. p. 566-80.

24. Schmid DM, Sauermann P, Werner M, Schuessler B, Blick N, Muentener M, et al. Experience with 100 cases treated with botulinum-A toxin injections in the detrusor muscle for idiopathic overactive bladder syndrome refractory to anticholinergics. J Urol 2006;17:177-85.

25. Goldman HB. Long-term outcome of the use of intravesical botulinum toxin for the treatment of overactive bladder (OAB). BJU Int 2013;111:8.
26. Veeratterapillay R, Harding C, Teo L, Vasdev N, Abroaf A, Dorkin $\mathrm{TJ}$, et al. Discontinuation rates and inter-injection interval for repeated intravesical botulinum toxin type? A injections for detrusor overactivity. Int J Urol 2014;21:175-8.

27. Leitner L, Guggenbuhl-Roy S, Knupfer SC, Walter M, Schneider MP, Tornic J, et al. More than 15 years of experience with intradetrusor onabotulinumtoxina injections for treating refractory neurogenic detrusor overactivity: lessons to be learned. Eur Urol 2016;70:522-8.

28. Laviana A, Jellison F, Kim JH. Sacral neuromodulation for refractory overactive bladder, interstitial cystitis, and painful bladder syndrome. Neurosurg Clin N Am 2014;25:33-46.

29. Amundsen CL, Richter HE, Menefee SA, Komesu YM, Arya LA, Gregory WT, et al. OnabotulinumtoxinA vs sacral neuromodulation on refractory urgency urinary incontinence in women: a randomized clinical trial. JAMA 2016;316:1366-74. 